\title{
PDF UNCERTAINTIES: A STRONG TEST OF GOODNESS OF FIT TO MULTIPLE DATA SETS
}

\author{
JOHN COLLINS* \\ DESY, Notkestraße 85, D-22603 Hamburg, Germany, \\ and \\ II Institut für Theoretische Physik, Universität Hamburg, \\ Luruper Chaussee 149, D-22761 Hamburg, Germany \\ E-mail: collins@phys.psu.edu \\ JON PUMPLIN \\ Department of Physics and Astronomy, \\ Michigan State University, East Lansing MI 48824, U.S.A. \\ E-mail: pumplin@pa.msu.edu
}

\begin{abstract}
We present a new criterion for the goodness of global fits. It involves an exploration of the variation of $\chi^{2}$ for subsets of data.
\end{abstract}

\section{Introduction}

This talk addresses the questions of quantifying how good global fits are, and of how we would know a theory is wrong; it summarizes our Ref. 1 .

The obvious criterion is that of hypothesis testing: $\chi^{2}=N \pm \sqrt{2 N}$ for a good fit with $N$ degrees of freedom. One should also apply the same criterion to subsets of data (e.g., from a particular experiment or reaction), for which the normal range is $\chi_{i}^{2}=N_{i} \pm \sqrt{2 N_{i}}$.

In fact, a much stronger criterion applies. The idea was discovered by contrasting the criteria for a one-standard-deviation effect in hypothesis testing and in parameter fitting. When fitting a single parameter, the one-sigma range of the parameter is found by increasing $\chi^{2}$ one unit above its minimum. But the fit is good (hypothesis-testing) at the one-sigma level if $\chi_{\min }^{2}$ is within $\sqrt{2 N}$ of its normal value $N$.

What is shown in Ref. 1 is that the goodness of a global fit is better tested by applying the parameter-fitting criterion in a certain way to subsets of data. This can be much more stringent than the obvious hypothesis-testing criterion.

*ON LEAVE FROM: PHYSICS DEPARTMENT, PENN STATE UNIVERSITY, 104 DAVEY LABORATORY, UNIVERSITY PARK PA 16802 U.S.A.

To appear in Proceedings of 9th International Workshop on Deep Inelastic Scattering and QCD (DIS 2001), Bologna, Italy, 27 Apr-1 May 2001 
Table 1. Hypothetical comparison of data and pdf's.

\begin{tabular}{|r|ccc|}
\hline PDF & $\begin{array}{c}\text { TEV } \\
(100 \mathrm{pts})\end{array}$ & $\begin{array}{c}\text { HERA } \\
(100 \mathrm{pts})\end{array}$ & $\begin{array}{c}\text { Total } \\
(200 \mathrm{pts})\end{array}$ \\
\hline CTEQ & 85 & 115 & 200 \\
MRST & 115 & 85 & 200 \\
\hline
\end{tabular}

\section{Scenario}

Suppose we have two pre-existing good global fits of parton densities, called CTEQ and MRST, and that new data arrive from two experiments, TEV and HERA. Assume that the $\chi^{2} \mathrm{~s}$ are as in Table 1, so that by the hypothesistesting criterion, each set of pdf's gives a good fit to each experiment.

In fact we may have a bad fit. This can be seen by constructing pdf's that interpolate between CTEQ and MRST, $f_{p}=p f^{\mathrm{CTEQ}}+(1-p) f^{\mathrm{MRST}}$, and then fitting the interpolating parameter $p$. If, for example, we have $\chi_{\mathrm{TEV}}^{2}=85+30(1-p)^{2}$ and $\chi_{\mathrm{HERA}}^{2}=85+30 p^{2}$, then the TEV data implies $p=1 \pm 0.18$, while the HERA data implies $p=0 \pm 0.18$.

By converting the problem to one of parameter fitting, we have found that the theory and experiments are mutually inconsistent in this case, by about $4 \sigma$. If the forms for $\chi^{2}$ are different, it is possible to have a good fit to both experiments, but only if neither CTEQ nor MRST fit the data. The decreases of 30 in $\chi^{2}$ between the CTEQ and MRST values are sufficient to show that at least one of these situations arises.

\section{General procedure and application to CTEQ5}

Observe that in the hypothetical scenario, CTEQ alone obtains a good $\chi^{2}$, and we only saw a problem when we brought in MRST. How can CTEQ alone determine that there is a problem without MRST's assistance, and vice versa? And how can this be done without knowing which of $\sim 30$ parameters is the important one?

The procedure we propose 1 is as follows:

- Take pre-determined subsets of data (e.g., experiments).

- Explore a region of parameter-space with $\chi^{2}-\chi_{\min }^{2}$ up to about $\sqrt{2 N}$, e.g., 50 for CTEQ/MRST, and find the minimum of $\chi_{i}^{2}$ for each subset.

- If $\min \left(\chi_{i}^{2}\right)-\chi_{i}^{2}$ (global fit) is less than a few units, then experiment $i$ disagrees with global fit. 


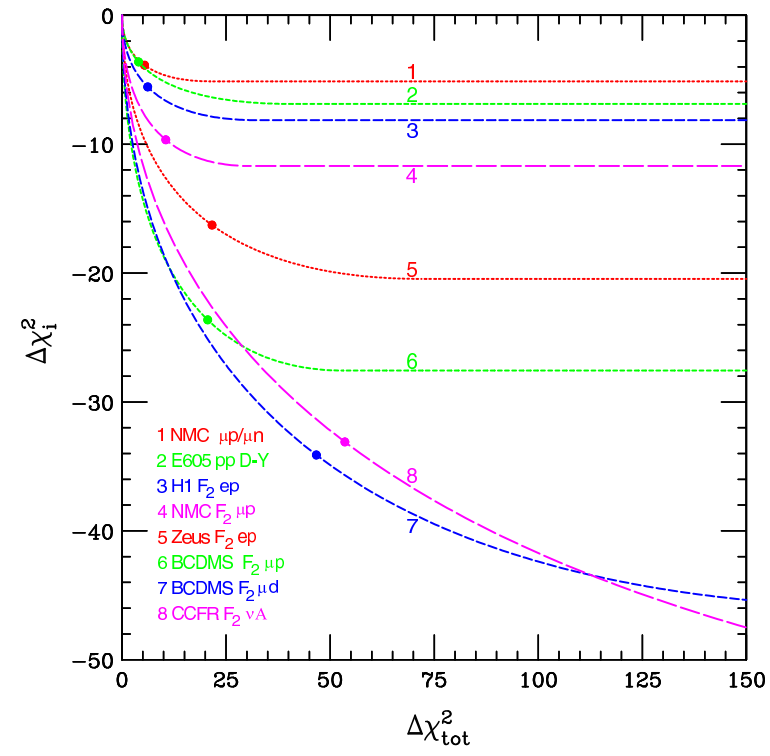

Figure 1. Application of our metpod to the 8 data sets that have the lion's share of the data points used in the CTEQ5 2 analysis. The values of $\Delta \chi^{2}$ plotted are the deviations of the $\chi^{2}$ from the values at the overall best fit.

Table 2. Values of $\chi^{2}$ for data contributing to the CTEQ5 fit.

\begin{tabular}{|l|rrrr|}
\hline Expt & $N$ & $\chi^{2}$ & $\chi^{2} / N$ & $\frac{\chi^{2}-N}{\sqrt{2 N}}$ \\
\hline 1. NMC D/H & 123 & 111 & 0.90 & -0.8 \\
2. E605 & 119 & 92 & 0.77 & -1.8 \\
3. H1 $F_{2}$ '96 & 172 & 108 & 0.63 & -3.4 \\
4. NMC H & 104 & 108 & 1.04 & 0.3 \\
\hline 5. ZEUS $F_{2}{ }^{\prime} 94$ & 186 & 249 & 1.34 & 3.3 \\
6. BCDMS H & 168 & 146 & 0.87 & $\mathbf{- 1 . 2}$ \\
7. BCDMS D & 156 & 222 & 1.42 & 3.7 \\
8. CCFR $F_{2}$ & 87 & 74 & 0.85 & $\mathbf{- 1 . 0}$ \\
\hline
\end{tabular}

- In computing significance, allow for the number of experiments and the effective number of parameters determined by each subset of data.

The minimization of $\chi_{i}^{2}$ for a given $\chi_{\text {total }}^{2}$ can be readily implemented by a Lagrange multiplier method: For each value of a parameter $\lambda$, minimize 
$\chi_{\text {tot }}^{2}(\mathbf{p})+(\lambda-1) \chi_{i}^{2}(\mathbf{p})$. The result gives a curve for $\chi_{i}^{2}$ as a function of $\chi_{\text {tot }}^{2}$.

The results of applying this procedure to the CTEQ5 fit are shown in Fig. 1. Several of the data sets can be seen to be bad fits, notably from CCFR and BCDMS. The criterion is that the $\chi^{2}$ for the subset of data decreases by too many units as $\chi_{\text {tot }}^{2}$ increases. A small decrease is normal and expected. The bad fit happens even though nothing exceptional happens according to the hypothesis-testing criterion, as can be seen in the last colımn of Table 2 .

MRST's plots of $\chi^{2}$ against $\alpha_{s}\left(M_{Z}\right)$ in Fig. 21 of Ref. 3 independently confirm our physics conclusion, that current data and QCD theory (including the approximations of NLO calculations, neglect of nuclear target and highertwist corrections, etc.) are not in good agreement.

\section{Conclusions}

We have shown that the quality of a global fit is correctly determined by testing the variation of $\chi^{2}$ (subset) for subsets of data as parameters are varied. A substantial decrease is a symptom of a bad fit, and the parameter-fitting criterion is the correct one here. With this method small data sets do not get lost compared with the other $\sim 1000$ points. The current CTEQ5 global fit appears not to fit the data, and the same appears to apply to the MRST fit.

Statistical analysis alone cannot tell us the explanation of this inconsistency. Only a physics-based analysis can decide if the problem is in one of the experiments, if there is a technical error in a theory calculation, or if there is really new physics that has been measured. The statistics only give a diagnosis of where further investigation will be most useful.

\section{Acknowledgments}

This work was supported in part by the U.S. Department of Energy and by the National Science Foundation. We would like to thank R. Thorne for conversations at this workshop. JCC would like to thank the Alexander von Humboldt foundation for an award.

\section{References}

1. J.C. Collins and J. Pumplin, "Tests of goodness of fit to multiple data sets", preprint DESY-01-014, hep-ph/0105207.

2. H.L. Lai et al. [CTEQ Collaboration], Eur. Phys. J. C12, 375 (2000).

3. A.D. Martin, R.G. Roberts, W.J. Stirling and R.S. Thorne, Eur. Phys. J. C4, 463 (1998). 